\title{
Density-based mixing parameter for hybrid functionals
}

\author{
Miguel A. L. Marques, ${ }^{1,2}$ Julien Vidal, ${ }^{1,3,2}$ Micael Oliveira, ${ }^{1,2}{ }^{2}$ Lucia Reining, ${ }^{3,2}$ and Silvana Botti ${ }^{3,1,2}$ \\ ${ }^{1}$ LPMCN, Université Claude Bernard Lyon I and CNRS, 69622 Villeurbanne, France \\ ${ }^{2}$ European Theoretical Spectroscopy Facility (ETSF) \\ ${ }^{3}$ Laboratoire des Solides Irradiés, École Polytechnique, CNRS, CEA-DSM, 91128 Palaiseau, France
}

\begin{abstract}
A very popular ab-initio scheme to calculate electronic properties in solids is the use of hybrid functionals in density functional theory (DFT) that mixes a portion of Fock exchange with DFT functionals. In spite of their success, a major problem still remains, related to the use of one single mixing parameter for all materials. Guided by physical arguments that connect the mixing parameter to the dielectric properties of the solid, and ultimately to its band gap, we propose a method to calculate this parameter from the electronic density alone. This method is able to cut significantly the error of traditional hybrid functionals for large and small gap materials, while retaining a good description of structural properties. Moreover, its implementation is simple and leads to a negligible increase of the computational time.
\end{abstract}

Density functional theory (DFT) is one of the major achievements of theoretical physics in the last decades. It is now routinely used to interpret experiments or to predict properties of novel materials. The success of DFT relies on the Kohn-Sham (KS) scheme and the existence of good approximations for the unknown exchange and correlation (xc) functional. In the standard KS formulation the xc potential is local and static. Since the original suggestion of the local-density approximation (LDA) [1], a swarm of functionals has been proposed in the literature [2]. In the ab-initio study of solids, the Perdew, Burke and Ernzerhof [5] (PBE) parametrization of the xc functional has been for many years the default choice for many applications. A good functional must yield ground states properties (like structural parameters), while it is expected that the KS gap and true quasiparticle gap differ by the derivative discontinuity [3]. Indeed, for semiconductors and insulators PBE yields good structural properties and KS band-gap energies that are at best half of their experimental value. To obtain both the ground state and quasiparticle energies correctly within one and the same formalism, one can resort to, e.g., a many-body $G W$ calculation [6, 7]. However, $G W$ is by all measures an expensive technique, with a very unfavorable scaling with the number of atoms in the unit cell. It is therefore unpractical for the study of band structures of large systems and clearly prohibitive regarding total energy calculations even for simple realistic systems.

Much of the computational effort in $G W$ comes from the dynamically screened Coulomb interaction $W$. It has therefore been crucial to explore to which extent dynamical effects are mandatory, or whether non-locality is the dominating characteristic. The move from local KS potential to non-local functionals has first been pushed forward in Quantum Chemistry, where today the so-called hybrid functionals are very popular. These functionals mix a fraction $\alpha$ of Fock exchange with a combination of LDA and generalized gradient (GGA) functionals. The application of hybrid functionals to the solid state had a much slower start [8, 9]. The situation changed re- cently, helped by the wider availability of computer codes that support hybrids [10] and the continued increase of computational power covering the additional cost with respect to a local potential. Besides yielding good structural properties [12] hybrids have proved to correct to a large extent the band-gap problem [9, 11]. Another landmark came with the introduction of screened hybrids [13]. These functionals lead to faster calculations and improved band-gaps, especially for small band-gap systems. Furthermore, by screening the Coulomb interaction at large distances, they also give access to metals.

The intuition lying behind an hybrid functional is rather clear. While LDA or GGA calculations strongly underestimate the gap, Hartree-Fock calculations overestimates it typically by more than a factor of two. By changing the mixing from 0 to 1 , one has a continuous change between local KS and Hartree-Fock, and an essentially linear variation between the respective gaps. Therefore, to obtain the correct experimental gap one simply has to use an appropriate mixing in the functional. This value can be determined from a fit to a series of systems, and set to around $\alpha \sim 0.2-0.3$. This choice gives very good results for a large class of systems, but it usually fails when the gap is very large or very small. But what is the physical meaning of the mixing parameter? To answer this question, it can be instructive to move away from a generalized KS picture 14], and consider the hybrid as an approximation to the self-energy $\Sigma$. In the GW approximation the latter can be written as

$$
\Sigma\left(\boldsymbol{r}, \boldsymbol{r}^{\prime} ; \omega\right)=\Sigma_{\mathrm{sX}}\left(\boldsymbol{r}, \boldsymbol{r}^{\prime}\right)+\Sigma_{\text {rest }}\left(\boldsymbol{r}, \boldsymbol{r}^{\prime} ; \omega\right),
$$

with $\Sigma_{\mathrm{SX}}\left(\boldsymbol{r}, \boldsymbol{r}^{\prime}\right)$ being the statically screened-exchange (sX) term, and $\Sigma_{\text {rest }}\left(\boldsymbol{r}, \boldsymbol{r}^{\prime} ; \omega\right)$ containing the static Coulomb hole and dynamical contributions. If the screening in the $\mathrm{sX}$ term is replaced by an effective static dielectric constant $\epsilon_{\infty}=1 / \alpha$, and $\Sigma_{\text {rest }}$ is modeled by the static and local part of the hybrid functional [15] the quasi-particle equation has the same form as the generalized KS equation solved for hybrid functionals 16]. From these arguments we can conclude that the physical value 


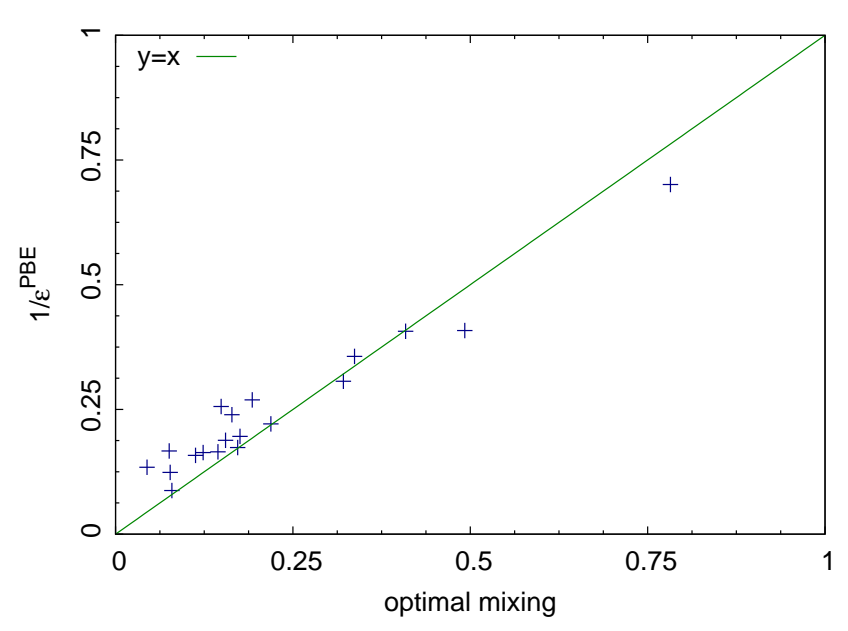

FIG. 1. (Color online) Inverse of the dielectric constant calculated with the PBE as a function of the optimal mixing parameter. The line $\alpha_{\mathrm{opt}}=1 / \epsilon_{\infty}^{\mathrm{PBE}}$ is a guide to the eye.

for the mixing parameter $\alpha$ is related to the inverse of the dielectric constant of the material at hand. Such a link has been suggested to explain also variations of band gaps with respect to small structural changes [18].

In Fig. 1 we show that, for a large range of different materials, the value of $1 / \epsilon_{\infty}$ - obtained with ABINIT [19] within the PBE approximation - is approximately proportional to the optimal mixing parameter $\alpha_{\text {opt }}$. The latter, calculated with the computer code VASP [10], is defined as the fraction $\alpha$ of Fock exchange of a PBE0 [8] hybrid functional that reproduces the experimental bandgap of the material. The correlation is evident, despite the fact that the DFT-PBE calculations systematically overestimate the dielectric constants with respect to experiment.

In Fig. 2 we compare the band-gaps calculated using $\alpha=1 / \epsilon_{\infty}^{\mathrm{PBE}}$ as the mixing parameter of a modified PBE0 hybrid functional (red stars labeled $\mathrm{PBE} 0 \epsilon_{\infty}$ ), compared to experimental data and other theoretical results. A more detailed comparison together with average errors can be found in the table given as supplemental materials. We can see that the hybrid PBE0 $(\alpha=1 / 4)[8]$ already improves dramatically the result with respect to a local PBE functional, bringing the calculated gaps towards the experimental values with an average error of less than a $30 \%$. However, this number alone hides the fact that PBE0 gives excellent results for some intermediate band gap materials, like diamond, BN, AlN, etc., whereas it fails both for small band gap materials (like $\mathrm{Si}$, or $\mathrm{Ge}$ ), overestimating their gaps, and for large band gap materials (like the rare-gases) where the gaps are underestimated. This is not surprising: In materials like Si, electrons are delocalized and easily polarizable, leading to a strong screening and small mixing (the optimal mixing is actually $\alpha_{\mathrm{opt}}=0.12$ ). For Ne, electrons are localized, and screening is basically nonexistent $\left(\alpha_{\text {opt }}=0.70\right)$. This effect is captured by the dependence on the dielectric constant of the mixing with $\alpha=1 / \epsilon_{\infty}$. Such a calculation decreases overall the error by almost a factor of two. The remaining error is dominated by the large underestimation of the gap for materials like Si, Ge, or GaAs. This point to the fact that, in order to predict good gaps approaching the metallic limit, one needs a finite amount of Fock exchange that is not accounted for by the simple $1 / \epsilon_{\infty}$ model for the mixing, as it can also be seen in Fig. 1.

The $\epsilon_{\infty}$-dependent mixing is hence physically motivated and can yield good band gaps, however, the procedure to obtain it (i.e. the calculation of the dielectric constant) is fairly cumbersome and therefore often unpractical. The best option would be to find an estimator for the dielectric constant from quantities readily available from the ground-state. To obtain such relation, we first observe that there is a strong correlation between the electronic dielectric constant of the material and the energy gap [20]. Of course, it is not desirable to have a functional depending explicitly on the band-gap of the material, so the question is if one can find an estimator of this quantity based solely on reduced densities.

In fact, several density-functional estimators of a "local gap" have been proposed in the past years. For example, Gutle et al. 21] proposed to use the quantity $G=\frac{1}{8}|\nabla n|^{2} / n^{2}$ to define the gap locally. Their arguments for the use of this quantity were based on the asymptotic expansion of the function $G$ for finite systems ( $G$ will reduce to the ionization energy) and on the observed piecewise exponential behavior of the density [22]. More recently, the quantity $|\nabla n| / n$ was used to model a position-dependent screening function in a so-called "local-hybrid" functional [13, 24]. Also the von Weizsäcker kinetic energy density $\tau_{\mathrm{W}}=|\nabla n|^{2} / 8 n$ has been used to define a "local band-gap", and inserted into a "local-hybrid" functional that turns off the exactexchange term when this local gap has metallic character [25].

To obtain a global estimator of the band-gap of the material, and therefore of its static dielectric constant, we can average the local estimator over the Brillouin zone. We will follow the idea contained in the meta-GGA of Tran and Blaha (TB09) 26] and define the quantity

$$
\bar{g}=\frac{1}{V_{\text {cell }}} \int_{\text {cell }} \mathrm{d}^{3} r \sqrt{\frac{|\nabla n(\boldsymbol{r})|}{n(\boldsymbol{r})}},
$$

where the integral is over the unit cell of volume $V_{\text {cell }}$. We note that the quantity $\bar{g}$ is very similar to the average involved in the calculation of the $c_{\mathrm{TB} 09}$ parameter of TB09, and is quite stable regardless on which theory is used to evaluate the density. In fact, we verified that using as input either a PBE or a Hartree-Fock density leads to only minor differences in its value. Our basic 

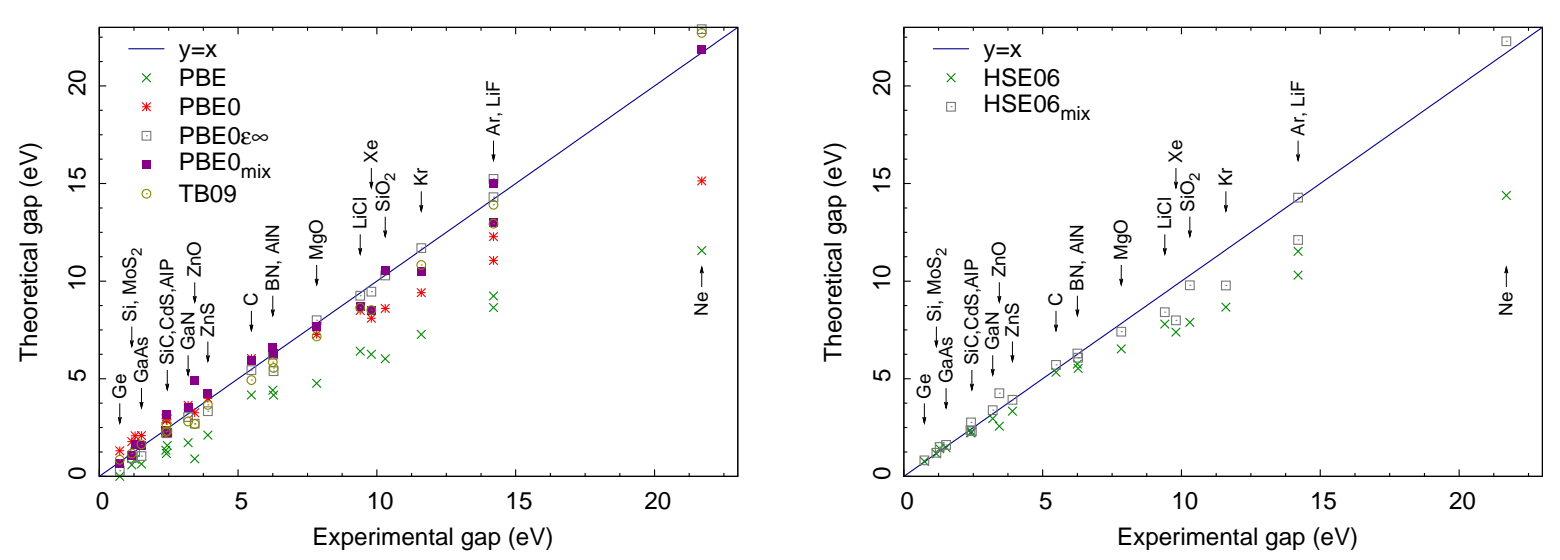

FIG. 2. (Color online) Electronic band-gaps calculated as differences of generalized KS eigenvalues for a series of semiconductors. All calculations were performed at the experimental lattice constant. The symbols labeled $\mathrm{PBE} 0_{\epsilon_{\infty}}, \mathrm{PBE} 0_{\text {mix }}$ and $\mathrm{HSE06}$ mix are the results obtained with the hybrid functionals proposed in this Article. TB09 results come from Ref. 26 .

hypothesis is that the mixing parameter can be written as a function of the parameter $\bar{g}$.

To proceed we need to specify the local part of the hybrid. We chose to use PBE0 form [8] which is the basis for the screened hybrid Heyd, Scuseria and Ernzerhof (HSE) [13]. As we expected, there is a clear correlation between the value of $\alpha_{\mathrm{opt}}$ and $\bar{g}$; it can be quite well fitted by the simple function $\alpha(\bar{g})$

$$
\alpha=-1.00778+1.10507 \bar{g}
$$

with $\bar{g}$ in atomic units.

Analyzing the resulting gaps displayed in Fig. 2 (labeled $P B E 0_{\text {mix }}$ ) we realize that fixing the mixing parameter according to Eq. 3. 23] reduces the mean average error to slightly more than $14 \%$, much better than PBE0, and slightly better than the HSE06 31] screened hybrid functional. Furthermore, in contrast with PBE0 and HSE06, our density-dependent mixing describes equally well small, medium and large gap systems. The largest errors arise for $d$-electron materials like $\mathrm{ZnO}$ where our recipe overestimates the mixing parameter and therefore the electronic gap. This is due to the fact that localized $d$-states give too large contributions to the mixing through strong density variations. A possible solution would be finding a more pertinent density-estimator for those $d$ states.

In view of the success of screened hybrids in improving the accuracy of PBE0, we applied our construction also to the HSE06 functional [31. In this case, the physical interpretation of the mixing parameter as an inverse screening is considerably more complicated, as screening is already present to some extent in the range separation. Following our protocol, we arrived at the following fit for the mixing parameter

$$
\alpha=0.121983+0.130711 \bar{g}^{4} .
$$

We remark the different power of $\bar{g}$ in the expression. This is due to the fact that the screening already present in HSE06 decreases considerably the strength of the Hartree-Fock term, increasing the values of $\alpha_{\text {opt }}$ required to reproduce the experimental band gap of small-gap systems. This is actually the cause of the success of HSE06: for example, for Si $\alpha_{\text {opt }}$ is now 0.24, which is very close to the actual mixing of HSE06 $(\alpha=1 / 4)$. Results for band-gaps using HSE06 and our new mixing scheme (labeled HSE06 mix ) are shown in Fig. 21). Our new mixing scheme brings down the HSE06 error from around $17 \%$ to $10 \%$, of the same order of magnitude as the error incurred by the $G_{0} W_{0}$ approximation and the new TB09 meta-GGA [26].

We want now to compare our approach to the the new TB09 meta-GGA. The physical interpretation of our functional implies that the mixing parameter should take values between 0 and 1 , while in TB09 the corresponding parameter $c_{\mathrm{TB} 09}$ is always larger than one. This difference stems from the fact that the TB09 functional is a purely local potential. Therefore, the band-gap defined in terms of total energy differences should be equal to the difference of the KS eigenvalues plus the derivative discontinuity of the xc potential. It is known that the Becke-Johnson potential 27] (upon which the TB09 functional has been constructed) reproduces to a very good extent the derivative discontinuity of exact exchange for molecular chains [28]. Therefore, it is reasonable to expect that the DFT band-gap with the TB09 functional, after adding the derivative discontinuity, would actually become much larger than experiment, and possibly even larger than the Hartree-Fock gap since $c_{\mathrm{TB} 09}$ is always larger than one, in agreement with what was proved by Grüning et al. 229]. Of course, the aim of TB09 is to obtain the gaps simply as eigenvalue differences, and therefore require $c_{\mathrm{TB} 09}>1$ in order to compensate for the un- 
derestimation of the eigenvalue gap in the Becke-Johnson functional [30]. In this sense, it is similar in spirit to the pragmatic $\mathrm{x} \alpha$ approach 32 ].

From Fig. 22 it is clear that the errors of TB09 is comparable to ours. On the one hand, the meta-GGA is clearly much lighter from the computational point of view than any hybrid functional. On the other hand, TB09 is an approximation for the xc potential, and is thus incapable of yielding total energies (in contrast to our approach). In fact, it can be proved that TB09 is not the functional derivative of any energy functional, and therefore violates serious constraints, like the zero-force theorem [33]. As a consequence, such functionals do not allow to calculate structural properties. Our approach, instead, can also be used to calculate total energies and structural properties. We tested our functionals and found that they give relaxed geometries as good as the standard PBE0 and HSE06 (with lattice constants better than $0.7 \%$ for the cubic semiconductors considered here). Moreover, we mention that the TB09 meta-GGA also inherited some of the problems of the Becke-Johnson functional [27] on which it is based (for example, TB09 is not size-consistent and gauge invariant [34]).

It is clear that the averaging procedure in Eq. (2) is only meaningful for bulk systems and will fail for finite systems, slabs, interfaces, etc. This issue can be fixed by converting the global $\bar{g}$ (and thus the uniform screening) into a local function function $\bar{g}(\mathbf{r})$, i.e. transforming the functional in a local hybrid. The function $\bar{g}(\mathbf{r})$ can be determined by restricting the integral of Eq. (2) to a neighborhood of $\mathbf{r}$, with a range related to the screening length of the system. This screening length can be taken as constant, as in the HSE functionals, or can be a local functional of the density (but clearly not a function of the average density). This procedure would not change significantly the results for the systems studied here as they all have very small unit cells.

In conclusion, we proposed a scheme to calculate onthe-fly the mixing parameter in hybrid functionals depending on the density of the system. In this way, the average error on the values of the energy gap were considerably reduced with respect to the original hybrid functionals. The resulting band-gaps are roughly of the same quality as those obtained using a $G W$ approach or the new meta-GGA of Tran and Blaha. Moreover, this method assures also an excellent description of the structural properties. These improvements are obtained with no increase of computational time with respect to a fixed mixing parameter.

Part of the calculations were performed at the LCA of the University of Coimbra and at GENCI (project x2010096017). We acknowledge funding from the European Commission through the e-I3 ETSF project (Contract \#211956). MALM, JV, and SB acknowledge partial funding from the French ANR (ANR-08-CEXC8-00801), and from the program PIR Matériaux - MaProSu of
CNRS.

[1] W. Kohn and L. J. Sham, Phys. Rev. 140, A1133 (1965).

[2] G. E. Scuseria and V.N. Staroverov, in: Theory and Applications of Computational Chemistry: The First Forty Years, ed. by C. E. Dykstra, G. Frenking, K. S. Kim, and G. E. Scuseria (Elsevier, Amsterdam, 2005), pp. 669-724.

[3] J. P. Perdew, R. G. Parr, M. Levy, and J. L. Balduz, Phys. Rev. Lett. bf 49, 1691 (1982); J. P. Perdew and M. Levy, Phys. Rev. Lett. 51, 1884 (1983); L. J. Sham and M. Schlüter, Phys. Rev. Lett. 51, 1888 (1983); Phys. Rev. B 32, 3883 (1985)

[4] P. J. Stephens, F. J. Devlin, C.F. Chabalowski, M. J. Frisch, J. Phys. Chem. 9811623 (1994).

[5] J. P. Perdew, K. Burke, and M. Ernzerhof, Phys. Rev. Lett. 77, 3865 (1996); ibid 78, 1396(E) (1997).

[6] L. Hedin, Phys. Rev. 139, A796 (1965).

[7] W. G. Aulbur and L. Jönsson and J. W. Wilkins, Solid State Phys. 54, 54 (2000).

[8] M. Ernzerhof, G. E. Scuseria, J. Chem. Phys. 110, 5029 (1999).

[9] E. N. Brothers, A. F. Izmaylov, J. O. Normand, V. Barone, and G. E. Scuseria, J. Chem. Phys. 129, 011102 (2008).

[10] G. Kresse and J. Furthmüller, Comput. Mater. Sci. 6, 15 (1996); G. Kresse and J. Furthmüller, Phys. Rev. B 54, 11169 (1996).

[11] J. Paier, M. Marsman and G. Kresse, Phys. Rev. B 78, 121201 (2008); J. Hafner, J. Comput. Chem. 29, 2044 (2008).

[12] J. Paier, M. Marsman and G. Kresse, J. Chem. Phys. 127, 024103 (2007).

[13] J. Heyd, G. E. Scuseria, and M. Ernzerhof, J. Chem. Phys. 118, 8207 (2003); ibid 124, 219906(E) (2006).

[14] A. Seidl, A. Görling, P. Vogl, J. A. Majewski, and M. Levy, Phys. Rev. B 53, 37641996.

[15] F. Gygi and A. Baldereschi, Phys. Rev. Lett. 62, 2160 (1989).

[16] Note that this is similar to the sX LDA method [14, 17], where $\Sigma$ is replaced by a Thomas-Fermi-screened exchange part and the LDA xc functional.

[17] D. M. Bylander and L. Kleinman, Phys. Rev. B 41, 7868 (1990)

[18] J. Vidal, S. Botti, P. Olsson, J-F. Guillemoles, and L. Reining Phys. Rev. Lett. 104, 056401 (2010).

[19] X. Gonze et al. , Z. Kristallogr. 220, 558 (2005).

[20] V. Fiorentini and A. Baldereschi, Phys. Rev. B 51, 17196 (1995).

[21] C. Gutle, A. Savin, J.B. Krieger, and J. Chen, Int. J. Quantum Chem. 75, 885 (1999).

[22] G. Sperber, Int. J. Quant. Chem., 5, 189 (1971); W.P. Wang, R. G. Parr, Phys. Rev. A 16, 891 (1977); M. Kohout, A. Savin, H. Preuss, J. Chem. Phys. 95, 1928 (1991).

[23] Note that in principle there is a contribution to the potential due to the variation of the mixing parameter $\alpha$ with the density. In these calculations we have neglected this term. However, we believe this to be a very good approximation due to the robustness of $\bar{g}$ to the actual details of the calculation of the density. 
[24] A. V. Krukau, G. E. Scuseria, J. P. Perdew, and A. Savin, J. Chem. Phys. 129, 124103 (2008).

[25] J. Jaramillo, G. E. Scuseria, M. Ernzerhof, J. Chem. Phys. 118, 1068 (2003).

[26] F. Tran and P. Blaha, Phys. Rev. Lett. 102, 226401 (2009).

[27] A. D. Becke and E. R. Johnson, J. Chem. Phys. 124, 221101 (2006).

[28] R. Armiento, S. Kümmel, and T. Körzdörfer, Phys. Rev. B 77, 165106 (2008).

[29] M. Grüning, A. Marini, and A. Rubio, J. Chem. Phys. 124, 154108 (2006).
[30] F. Tran, P. Blaha, and K. Schwarz, J. Phys. Condens. Matter 19, 196208 (2007).

[31] A. V. Krukau, O. A. Vydrov, A. F. Izmaylov, and G. E. Scuseria, J. Chem. Phys. 125, 224106 (2006). Note that a local-hybrid functional is a non-local functional.

[32] J. C. Slater Phys. Rev. 81, 385 (1951).

[33] A. P. Gaiduk and V. N. Staroverov, J. Chem. Phys. 131, 044107 (2009); A. Karolewski, R. Armiento, and S. Kummel, J. Chem. Theory Comput. 5, 712 (2009).

[34] E. Räsänen, S. Pittalis, and C. R. Proetto, Phys. Rev. B 81, 195103 (2010). 
TABLE I. Electronic band-gaps calculated as differences of Kohn-Sham eigenvalues for a series of semiconductors. All calculations were performed at the experimental lattice constant. The column label HF+c denotes Hartree-Fock including PBE correlation. The columns PBE $\epsilon_{\infty}, \mathrm{PBE} 0_{\text {mix }}$ and HSE06 mix present the results obtained with the hybrid functionals proposed in this Article. The columns TB09 and $G_{0} W_{0}$ are from [F. Tran and P. Blaha, Phys. Rev. Lett. 102, 226401 (2009)].

\begin{tabular}{|c|c|c|c|c|c|c|c|c|c|c|}
\hline & p. & $\mathrm{BE}$ & $\mathrm{F}+$ & & & & & & & \\
\hline $\mathrm{Ne}$ & 21.70 & 11.57 & 26.14 & 15.14 & 22.95 & 21.88 & 14.39 & 22.29 & 22.72 & 19.59 \\
\hline $\mathrm{Ar}$ & 14.20 & 8.65 & 18.45 & 11.06 & & 12.98 & 10.31 & 12.11 & 13.91 & 13.28 \\
\hline $\mathrm{Kr}$ & 11.60 & 7.27 & 16.04 & 9.41 & 11.75 & 10.48 & 8.67 & 9.78 & 10.83 & \\
\hline $\mathrm{Xe}$ & 9.80 & 6.25 & 13.79 & 8.10 & 9.5 & & 7.39 & 7.99 & 8.52 & \\
\hline $\mathrm{C}$ & 5.48 & 4.17 & 12.05 & 6.06 & & & 5.33 & & 4.93 & 5.50 \\
\hline $\mathrm{Si}$ & 1.17 & 0.59 & & & & & & & 1.17 & 1.12 \\
\hline $\mathrm{Ge}$ & 0.7 & 0. & & & & & & & 0.85 & 66 \\
\hline $\mathrm{Li}$ & 14.2 & 9.24 & 21. & 12.26 & 15 & 14 & 11 & 14 & 12.94 & 13.27 \\
\hline $\mathrm{Li}$ & & 6.41 & 14. & & & & & & 8.64 & \\
\hline $\mathrm{M}_{\varepsilon}$ & 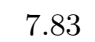 & 4.77 & 15. & & & & & & 7.17 & 7.25 \\
\hline $\mathrm{Si}$ & 2 . & 1.34 & & & & & & & 2.28 & 2.27 \\
\hline $\mathrm{BN}$ & 6.25 & 4.41 & 13.0 & & & & & 9 & 5.85 & 6.10 \\
\hline GaN & 3.2 & 1.72 & 10.29 & & & & & & 2.81 & 2.80 \\
\hline GaAs & 1.52 & 0.63 & & 9 & & 1 & 1.47 & 1 & 1.64 & 1.30 \\
\hline $\mathrm{AlP}$ & 2.45 & 1.58 & 7.40 & 2.93 & & 2.2 & 2.27 & 2.32 & 2.32 & 2.44 \\
\hline $\mathrm{ZnS}$ & 3.91 & 2.11 & 10.06 & 4.00 & & 4.2 & 3.34 & 3.92 & 3.66 & 3.29 \\
\hline CdS & 2.42 & 1.17 & & & 2.2 & & 2.23 & 2.76 & 2.66 & 2.06 \\
\hline AlI & 6.28 & 4.16 & 12.94 & 6.25 & 5.3 & 6.2 & 5. & 6.08 & 5.55 & 5.83 \\
\hline $\mathrm{SiO}_{2}$ & 10.30 & 6.02 & 16.7 & 8 & 9.1 & 10. & 7.8 & 9.7 & & \\
\hline $\mathrm{MoS}_{2}$ & 1.29 & 0.87 & & & & & & 1.5 & & \\
\hline $\mathrm{ZnO}$ & 3.44 & 0.90 & 11.21 & 3.26 & 2.74 & 4.90 & 2.57 & 4.26 & 2.68 & 2.51 \\
\hline $4(1 / 0$ & & 47.32 & 250.23 & 29.42 & 16.53 & 14.37 & 16.92 & 10.36 & 9.85 & 11.25 \\
\hline
\end{tabular}

